\title{
Approximate Solution of Wave Equation using Fuzzy Number
}

\author{
M. Aylin Bayrak \\ University of Kocaeli \\ Department of Mathematics \\ Kocaeli, Turkey
}

\begin{abstract}
In this paper, the fuzzy solution of the initial boundary value problem of hyperbolic one-dimensional wave equation is considered. The solution by finite difference method is observed by using fuzzy intervals.
\end{abstract}

\section{Keywords:}

$\alpha$-cut, fuzzy membership function, triangular fuzzy number, fuzzy interval

\section{INTRODUCTION}

In the context of fuzzy theory, first introduced by Zadeh [1], the arithmetic operations on fuzzy numbers are usually approached

either by the use of the extension principle (in the domain of the membership function) or by the interval arithmetics (in the domain of the $\alpha$-cuts). The exact analytical fuzzy operations dates back from the early 1980s and are outlined by Dubois and Prade [2] ; the same authors have introduced the well-known L-R model and the corresponding formulas for the fuzzy operations [3]. The fuzzy

differential equations and fuzzy initial value problem were regularly treated by Kaleva $[4,5]$. The numerical methods for

solving fuzzy differential equations are introduced in $[6,7,8,9]$. In this paper, first finite difference method to solve onedimensional wave equation is applied using fuzzy interval arithmetics

$[10,11,12]$, numerically.

\section{BASIC CONCEPT OF FUZZY SET THEORY}

A triangular fuzzy number $\mathrm{u}$ can be defined as a triplet $[a, b, c]$. Its membership function is defined as:

$$
\mu_{u}(x)= \begin{cases}\frac{x-a}{b-a}, & a \leq x \leq b \\ \frac{c-x}{c-b}, & b \leq x \leq c\end{cases}
$$

To find the $\alpha$-cut of $\mathrm{u}, \alpha \epsilon[0,1]$ to both left and right reference functions of $\mathrm{u}$ is set. That is, $\alpha=\frac{x-a}{b-a}$ and $\alpha=\frac{c-x}{c-b}$. Expressing $\mathrm{x}$ in terms of $\alpha, x=(b-a) \alpha+a$ and $x=c-(c-b) \alpha$ is obtained, which gives the $\alpha$-cut of $\mathrm{u}$ is $u_{\alpha}=[(b-a) \alpha+a, c-(c-b) \alpha]$.

If $u=\left[u^{-}, u^{+}\right]$and $v=\left[v^{-}, v^{+}\right]$are two given fuzzy numbers, the arithmetic operations are defined for $0 \leq \alpha \leq 1$ as follows:

1. $u=v$ if and only if $u_{\alpha}^{-}=v_{\alpha}^{-}$and $u_{\alpha}^{+}=v_{\alpha}^{+}$.

2. $u+v=\left[u_{\alpha}^{-}+v_{\alpha}^{-}, u_{\alpha}^{+}+v_{\alpha}^{+}\right]$.

3. $k u=\left[\min \left\{k u_{\alpha}^{-}, k u_{\alpha}^{+}\right\}, \max \left\{k u_{\alpha}^{-}, k u_{\alpha}^{+}\right\}\right],(k \epsilon \Re)$

$$
\text { 4. } u+v=\left[u_{\alpha}^{-}-v_{\alpha}^{+}, u_{\alpha}^{+}-v_{\alpha}^{-}\right] \text {. }
$$

\section{APPLICATION OF FINITE DIFFERENCE METHOD IN WAVE EQUATION}

Consider the initial boundary value problem of hyperbolic one-dimensional wave equation [13]

$$
\frac{\partial^{2} u}{\partial t^{2}}=a^{2} \frac{\partial^{2} u}{\partial x^{2}}, 0 \leq x \leq L, t \geq 0
$$

initial condition

$$
u(x, 0)=f(x)
$$

subject to boundary conditions

$$
u(0, t)=u(L, t)=0
$$

with initial velocity

$$
u_{t}(x, 0)=f(x) .
$$

Using finite difference method and substituting

$$
\begin{aligned}
& u_{t t}=\frac{1}{k^{2}}\left(u_{i, j+1}-2 u_{i, j}+u_{i, j-1}\right) \text { and } \\
& u_{x x}=\frac{1}{h^{2}}\left(u_{i+1, j}-2 u_{i, j}+u_{i-1, j}\right) \\
& \text { we get } \\
& \qquad u_{i, j+1}=r u_{i+1, j}+(2-2 r) u_{i, j}+r u_{i-1, j}-u_{i, j-1}
\end{aligned}
$$

where $r=\frac{a^{2} k^{2}}{h^{2}}$.This explicit method converges and stable for $0<r \leq 1$. For a given $a^{2}$, choose $\mathrm{h}$ and $\mathrm{k}$ such that $r=1$. Hence, (5) becomes

$$
u_{i, j+1}=u_{i+1, j}+u_{i-1, j}-u_{i, j-1} .
$$

For the initial condition $u_{t}(x, 0)=f(x)$, central difference approximation for the derivative is used and $u_{i 1}=\frac{f_{i+1}+f_{i-1}}{2}$ is written.

\section{FUZZIFICATION OF WAVE EQUATION}

Let $\quad u_{i-1, j-1}=\left[W_{01}, W_{02}, W_{03}\right] \quad$ and $u_{i+1, j-1}=\left[W_{21}, W_{22}, W_{23}\right]$.

$\left[u_{i, j}\right]=\left[\frac{W_{01}+W_{21}}{2}, \frac{W_{02}+W_{22}}{2}, \frac{W_{03}+W_{23}}{2}\right]=\left[E_{11}, E_{12}, E_{13}\right]$ is obtained. Firstly, fuzzy membership function for $u_{i-1, j-1}^{2}$ is

$$
\mu_{u_{i-1, j-1}}(X)= \begin{cases}\frac{X-W_{01}}{W_{02}-W_{01}}, & W_{01} \leq X \leq W_{02} \\ \frac{W_{03}-X}{W_{03}-W_{02}}, & W_{02} \leq X \leq W_{03}\end{cases}
$$

Then, $\left[u_{i-1, j-1}\right]_{\alpha}=\left[W_{01}+\left(W_{02}-W_{01}\right) \alpha, W_{03}-\left(W_{03}-\right.\right.$ $\left.\left.W_{02}\right) \alpha\right]$ is the $\alpha$-cut of fuzzy number $u_{i-1, j-1}$. 
Secondly, fuzzy membership function for $u_{i+1, j-1}$ is

$$
\mu_{u_{i+1, j-1}}(X)= \begin{cases}\frac{X-W_{21}}{W_{22}-W_{21}}, & W_{21} \leq X \leq W_{22} \\ \frac{W_{23}-X_{12}}{W_{23}-W_{22}}, & W_{22} \leq X \leq W_{23}\end{cases}
$$

Then, $\left[u_{i+1, j-1}\right]_{\alpha}=\left[W_{21}+\left(W_{22}-W_{21}\right) \alpha, W_{23}-\left(W_{23}-\right.\right.$ $\left.\left.W_{22}\right) \alpha\right]$ is the $\alpha$-cut of fuzzy number $u_{i+1, j-1}$.

Hence $\alpha$-cut for $u_{i, j}$ is $[14]$

$\left[u_{i, j}\right]_{\alpha}=\frac{1}{2}\left[\left\{\left(W_{01}+W_{21}\right)+\left\{\left(W_{02}-W_{01}\right)+\left(W_{22}-W_{21}\right)\right\} \alpha\right.\right.$,

Let

$X_{1}=\frac{\left\{\left(W_{01}+W_{21}\right)+\left\{\left(W_{02}-W_{01}\right)+\left(W_{22}-W_{21}\right)\right\} \alpha\right.}{2}$

$\Rightarrow \alpha=\frac{2 X_{1}-\left(W_{01}+W_{21}^{2}\right)}{\left(W_{02}-W_{01}\right)+\left(W_{22}-W_{21}\right)}$

and

$$
\begin{aligned}
& X_{2}=\frac{\left\{\left(W_{03}+W_{23}\right)-\left\{\left(W_{03}-W_{02}\right)+\left(W_{23}-W_{22}\right)\right\} \alpha\right.}{2} \\
& \Rightarrow \alpha=\frac{2 X_{2}-\left(W_{03}+W_{23}\right)}{\left(W_{03}-W_{02}\right)+\left(W_{23}-W_{22}\right)} .
\end{aligned}
$$

Thus, fuzzy membership function for $u_{i, j}$ is $\mu_{u_{i, j}}(X)=$

$\begin{cases}\frac{2 X-\left(W_{01}+W_{21}\right)}{\left(W_{02}+W_{22}\right)-\left(W_{01}+W_{21}\right)}, & \frac{\left(W_{01}+W_{21}\right)}{2} \leq X \leq \frac{\left(W_{02}+W_{22}\right)}{2} \\ \frac{-2 X+\left(W_{03}+W_{23}\right)}{\left(W_{03}+W_{23}\right)-\left(W_{02}+W_{22}\right)}, & \frac{\left(W_{02}+W_{22}\right)}{2} \leq X \leq \frac{\left(W_{03}+W_{23}\right)}{2}\end{cases}$

Now,let $u_{i-1, j}=\left[E_{01}, E_{02}, E_{03}\right], u_{i+1, j}=\left[E_{21}, E_{22}, E_{23}\right]$ and $u_{i, j-1}=\left[W_{11}, W_{12}, W_{13}\right]$. We get

$\left[u_{i, j+1}\right]=\left[E_{01}+E_{21}-W_{11}, E_{02}+E_{22}-W_{12}, E_{03}+E_{23}-\right.$ $\left.W_{13}\right]$. Firstly, fuzzy membership function for $u_{i-1, j}$ is

$$
\mu_{u_{i-1, j}}(X)= \begin{cases}\frac{X-E_{01}}{E_{02}-E_{01}}, & E_{01} \leq X \leq E_{02} \\ \frac{E_{03}-X}{E_{03}-E_{02}}, & E_{02} \leq X \leq E_{03}\end{cases}
$$

and $\left[u_{i-1, j}\right]_{\alpha}=\left[E_{01}+\left(E_{02}-E_{01}\right) \alpha, E_{03}-\left(E_{03}-E_{02}\right) \alpha\right]$ is the $\alpha$-cut of fuzzy number $u_{i-1, j}$.

Then, fuzzy membership function for $u_{i+1, j}$ is

$$
\mu_{u_{i+1, j}}(X)= \begin{cases}\frac{X-E_{21}}{E_{22}-E_{21}}, & E_{21} \leq X \leq E_{22} \\ \frac{E_{23}-X}{E_{23}-E_{22}}, & E_{22} \leq X \leq E_{23}\end{cases}
$$

and $\left[u_{i+1, j}\right]_{\alpha}=\left[E_{21}+\left(E_{22}-E_{21}\right) \alpha, E_{23}-\left(E_{23}-E_{22}\right) \alpha\right]$ is the $\alpha$-cut of fuzzy number $u_{i-1, j}$.

Then, fuzzy membership function for $u_{i, j-1}$ is

$$
\mu_{u_{i, j-1}}(X)= \begin{cases}\frac{X-W_{11}}{W_{12}-W_{11}}, \quad W_{11} \leq X \leq W_{12} \\ \frac{W_{13}-X}{W_{13}-W_{12}}, \quad W_{12} \leq X \leq W_{13}\end{cases}
$$

and $\left[u_{i, j-1}\right]_{\alpha}=\left[W_{11}+\left(W_{12}-W_{11}\right) \alpha, W_{13}-\left(W_{13}-W_{12}\right) \alpha\right]$ is the $\alpha$-cut of fuzzy number $u_{i, j-1}$.

Hence, $\alpha$-cut for $u_{i, j+1}$ is $\left[u_{i, j+1}\right]_{\alpha}=$

$\left[\left(E_{01}+E_{21}-W_{13}\right)+\left\{\left(E_{02}-E_{01}\right)+\left(E_{22}-E_{21}\right)+\left(W_{13}-\right.\right.\right.$ $\left.\left.W_{12}\right)\right\} \alpha,\left(E_{03}+E_{23}-W_{11}\right)-\left\{\left(E_{03}-E_{02}\right)+\left(E_{23}-E_{22}\right)+\right.$ $\left.\left.\left(W_{12}-W_{11}\right)\right\} \alpha\right]$.

Let

$X_{1}=$

$\left(E_{01}+E_{21}-W_{13}\right)+\left\{\left(E_{02}+E_{22}-W_{12}\right)-\left(E_{01}+E_{21}-W_{13}\right)\right\} \alpha$

$\Rightarrow \alpha=\frac{X_{1}-\left(E_{01}+E_{21}-W_{13}\right)}{\left(E_{02}+E_{22}-W_{12}\right)-\left(E_{01}+E_{21}-W_{13}\right)}$

and

$X_{2}=$

$\left(E_{03}+E_{23}-W_{11}\right)-\left\{\left(E_{03}+E_{23}-W_{11}\right)-\left(E_{02}+E_{22}-W_{11}\right)\right\} \alpha$ $\Rightarrow \alpha=\frac{-X_{2}+\left(E_{03}+E_{23}-W_{11}\right)}{\left(E_{03}+E_{23}-W_{11}\right)-\left(E_{02}+E_{22}-W_{11}\right)}$.

Then, fuzzy membership function for $u_{i, j+1}$ is on the way

$A=E_{01}+E_{21}-W_{13}, B=E_{02}+E_{22}-W_{12}$ and

$C=E_{03}+E_{23}-W_{11}$

$$
\mu_{u_{i, j+1}}(X)= \begin{cases}\frac{X-A}{B-A}, & A \leq X \leq B \\ \frac{C-X}{C-B}, & B \leq X \leq C\end{cases}
$$

With the help of this fuzzy membership function of different u's of $j+1$ th level can be found out.

\section{NUMERICAL EXAMPLES}

Consider the one-dimensional wave equation $\frac{\partial^{2} u}{\partial t^{2}}=16 \frac{\partial^{2} u}{\partial x^{2}}$ with the conditions $u(x, 0)=x^{2}(5-x)$ $u(0, t)=u(5, t)=0$ $u_{t}(x, 0)=0$

Let $u_{00}=[-0.001,0,0.001]$ and $u_{20}=[11.999,12,12.001]$. and $u_{11}=[5.999,6,6.001]$.

Firstly, fuzzy membership function for $u_{00}$ is

$$
\mu_{u 00}(x)= \begin{cases}\frac{x+0.001}{0.001}, & -0.001 \leq x \leq 0 \\ \frac{12.001-x}{0.001}, & 0 \leq x \leq 0.001\end{cases}
$$

and $\alpha$-cut for $u_{00}$ is $\left[u_{00}\right]_{\alpha}=[0.001 \alpha-0.001,0.001-0.001 \alpha]$ Secondly, fuzzy membership function for $u_{20}$ is

$$
\mu_{u 20}(x)= \begin{cases}\frac{x-11.999}{0.001}, & 11.999 \leq x \leq 12 \\ \frac{12.001-x}{0.001}, & 12 \leq x \leq 12.001\end{cases}
$$

and $\alpha$-cut for $u_{20}$ is $\left[u_{20}\right]_{\alpha}=[0.001 \alpha+11.999,12.001-$ $0.001 \alpha]$. Thus, $\alpha$-cut for $u_{11}$ is $\left[u_{11}\right]_{\alpha}=[0.001 \alpha+$ $5.999,6.001-0.001 \alpha]$. Let $\quad X_{1}=5.999+0.001 \alpha \Rightarrow \alpha=$ $\frac{X_{1}-5.999}{0.001}$ and

$X_{2}=6.001-0.001 \alpha \Rightarrow \alpha=\frac{-X_{2}+6.001}{0.001}$.

Hence, fuzzy membership function for $u_{11}$ is

$$
\mu_{u 11}(x)= \begin{cases}\frac{x-5.999}{0.001}, & 5.999 \leq x \leq 6 \\ \frac{6.001-x}{0.001}, & 6 \leq x \leq 6.001\end{cases}
$$

Now, let $u_{01}=[-0.001,0,0.001], u_{21}=[10.999,11,11.001]$, $u_{10}=[3.999,4,4.001]$. and $u_{12}=[6.997,7,7.003]$. Firstly, fuzzy membership function for $u_{01}$ is

$$
\mu_{u 01}(x)= \begin{cases}\frac{x+0.001}{0.001}, & -0.001 \leq x \leq 0 \\ \frac{0.001-x}{0.001}, & 0 \leq x \leq 0.001\end{cases}
$$

and $\left[u_{01}\right]_{\alpha}=[0.001 \alpha-0.001,0.001-0.001 \alpha]$. Then, fuzzy membership function for $u_{21}$ is

$$
\mu_{u 21}(x)= \begin{cases}\frac{x-10.999}{10.001-x}, & 10.999 \leq x \leq 11 \\ \frac{11.01-001}{0.01}, & 11 \leq x \leq 11.001\end{cases}
$$

and $\left[u_{21}\right]_{\alpha}=[0.001 \alpha+10.999,11.001-0.001 \alpha]$. Then, fuzzy membership function for $u_{10}$ is

$$
\mu_{u 10}(x)= \begin{cases}\frac{x-3.999}{0.001-x}, & 3.999 \leq x \leq 4 \\ \frac{4.001-001}{0.001}, & 4 \leq x \leq 4.001\end{cases}
$$

and $\left[u_{10}\right]_{\alpha}=[0.001 \alpha+3.999,4.001-0.001 \alpha]$. Thus, $\left[u_{12}\right]_{\alpha}=[0.003 \alpha+6.997,7.003-0.003 \alpha]$. Let $X_{1}=6.997+0.003 \alpha \Rightarrow \alpha=\frac{X_{1}-6.997}{0.003}$ and $X_{2}=7.003-0.003 \alpha \Rightarrow \alpha=\frac{-X_{2}+7.003}{0.003}$. Hence, fuzzy membership function for $u_{12}$ is

$$
\mu_{u 12}(x)= \begin{cases}\frac{x-6.997}{0.003}, & 6.997 \leq x \leq 7 \\ \frac{7.003-x}{0.003}, & 7 \leq x \leq 7.003\end{cases}
$$

Similarly, fuzzy membership functions can be found out at different grid points. In Table 1-2, the $\alpha$-cut with left and right functions of fuzzy number $\mathrm{u}$ is shown for $\alpha=0$, respectively.

\section{CONCLUSION}

In this paper, fuzzy membership functions to find solution of wave equation has been shown in numerical fuzzified form. $\mathrm{Nu}-$ merical computations have been made to illustrate ability and reliability of the method. 
Table 1. The left side of fuzzy number $u$ for $\alpha=0$.

\begin{tabular}{|c|c|c|c|c|c|c|}
\hline $\mathrm{j} \backslash i$ & 0 & 1 & 2 & 3 & 4 & 5 \\
\hline 0 & -0.001 & 3.999 & 11.999 & 17.999 & 15.999 & -0.001 \\
\hline 1 & -0.001 & 5.999 & 10.999 & 13.999 & 8.999 & -0.001 \\
\hline 2 & -0.001 & 6.997 & 7.997 & 1.997 & -2.003 & -0.001 \\
\hline 3 & -0.001 & 1.995 & -2.007 & -8.007 & -7.005 & -0.001 \\
\hline 4 & -0.001 & -9.011 & -14.015 & -11.015 & -6.011 & -0.001 \\
\hline 5 & -0.001 & -16.021 & -18.033 & -12.033 & -4.021 & -0.001 \\
\hline 6 & -0.001 & -9.035 & -14.069 & -11.069 & -6.045 & -0.001 \\
\hline 7 & -0.001 & 1.909 & -2.137 & -8.147 & -7.091 & -0.001 \\
\hline 8 & -0.001 & 6.817 & 7.693 & 1.703 & -2.193 & -0.001 \\
\hline 9 & -0.001 & 5.601 & 10.373 & 13.353 & 8.611 & -0.001 \\
\hline 10 & -0.001 & 3.189 & 10.647 & 16.677 & 15.159 & -0.001 \\
\hline
\end{tabular}

Table 2. The right side of fuzzy number u for $\alpha=0$.

\begin{tabular}{|c|c|c|c|c|c|c|}
\hline $\mathrm{j} \backslash i$ & 0 & 1 & 2 & 3 & 4 & 5 \\
\hline 0 & 0.001 & 4.001 & 12.001 & 18.001 & 16.001 & 0.001 \\
\hline 1 & 0.001 & 6.001 & 11.001 & 14.001 & 9.001 & 0.001 \\
\hline 2 & 0.001 & 7.003 & 8.003 & 2.003 & -1.997 & 0.001 \\
\hline 3 & 0.001 & 2.005 & -1.993 & -7.993 & -6.995 & 0.001 \\
\hline 4 & 0.001 & -8.989 & -13.985 & -10.985 & -5.989 & 0.001 \\
\hline 5 & 0.001 & -15.979 & -17.967 & -11.967 & -3.979 & 0.001 \\
\hline 6 & 0.001 & -8.955 & -13.931 & -10.931 & -5.955 & 0.001 \\
\hline 7 & 0.001 & 2.091 & -1.853 & -7.853 & -6.909 & 0.001 \\
\hline 8 & 0.001 & 7.183 & 8.307 & 2.307 & -1.807 & 0.001 \\
\hline 9 & 0.001 & 6.399 & 11.627 & 14.647 & 9.399 & 0.001 \\
\hline 10 & 0.001 & 5.811 & 13.353 & 19.323 & 15.841 & 0.001 \\
\hline
\end{tabular}

\section{REFERENCES}

[1]. Zadeh L.A., Fuzzy Sets, Inform. Control 8 (1965)338-353. [2]. Dubois D., Prade H., Fuzzy Sets and Systems. Theory and Applications, Academic Press, New York,1980.
[3]. Dubois D., Prade H., Possibility Theory. An approach to computerized processing of uncertainty, Plenum Press, New York, 1988.

[4]. Kaleva O., Fuzzy Differential Equations, Fuzzy sets and systems 24 (1987)301-317.

[5]. Kaleva O., The cauchy problem for fuzzy differential equations, Fuzzy sets and systems 35 (1990)389-396.

[6]. Abbasbandy S., Allahviranloo T., Numerical solution of fuzzy differential equations by Taylor method, Journal of Computational Methods in Applied Mathematics 2 (2002)113-124.

[7]. Abbasbandy S., Allahviranloo T., O. Lopez-Pouso, J.J.Nieto, Numerical methods for fuzzy differential inclusions, Journal of Computer and Mathematics with Applications 48 (2004)16331641.

[8]. Friedman M., Ma M., Kandel A., Numerical solutions of fuzzy differential and integral equations, Fuzzy sets and systems 106 (1999)35-48

[9]. Ma M., Friedman M., Kandel A., Numerical solutions of fuzzy differential equations, Fuzzy sets and systems 86 (1997) 331-334.

[10]. Kaufman A., Gupta M.M., Introduction to fuzzy arithmetic, Theory and applications, Van Nostrand Reinhold Co. Inc., Workingham, Berkshire, 1984.

[11]. Dutta P., Boruah H., Ali T., Fuzzy arithmetic with and without using $\alpha$-cut method: A comparative study, International Journal of Latest trends in computing 2(1) (2011)99-107.

[12]. Guerra M.L., Stefanini L., Approximate fuzzy arithmetic operations using monotonic interpolants, Fuzzy sets and systems 150 (2005)5-33.

[13]. Ramana B.V., Higher Engineering Mathematics, McGraw Hill Publishing Company, New Delhi, 2008.

[14]. Saikia R.K., Fuzzification of heat equation, International journal of computer applications 46(21) (2012)23-26. 